Pacific Journal of Mathematics

AN EXTENSION OF A THEOREM OF JORDAN AND YON 


\title{
AN EXTENSION OF A THEOREM OF JORDAN AND VON NEUMANN
}

\author{
LEONARD M. BLUMENTHAL
}

1. Introduction. Let $\{\mathbf{E}\}$ denote the class of generalized euclidean spaces $\mathbf{E}$ ( that is, $\mathbf{E} \subseteq\{\mathbf{E}\}$ provided all finite dimensional subspaces of $\mathbf{E}$ are euclidean spaces). The problem of characterizing metrically the class $\{\mathbf{F}\}$ with respect to the class $\{\mathbf{B}\}$ of all Banach spaces has been solved in many different ways. ${ }^{1}$ Fréchet's characteristic conditions [5]

$$
\frac{1}{2} \sum_{i, j=1}^{3}\left[\left\|p_{i}\right\|^{2}+\left\|p_{j}\right\|^{2}-\left\|p_{i}-p_{j}\right\|^{2}\right] x_{i} x_{j} \geq 0, \quad\left(p_{1}, p_{2}, p_{3} \in \mathbf{B}\right)
$$

was immediately we akened by Jordan and von Neumann [6] to

$$
\left\|p_{1}+p_{2}\right\|^{2}+\left\|p_{1}-p_{2}\right\|^{2}=2\left(\left\|p_{1}\right\|^{2}+\left\|p_{2}\right\|^{2}\right) \quad\left(p_{1}, p_{2} \in \mathbf{B}\right) .
$$

This relation has now become a kind of standard to which others repair by show* ing that it is implied by newly postulated conditions $[3,4,10]$, and it has been, apparently, the motivation of work in which it does not enter directly $[7,9]$. Perhaps the best possible result in this direction, however, is due to Aronszajn [1] who assumed merely that

$$
\|(x+y) / 2\|=\frac{1}{2} \phi(\|x\|,\|y\|,\|x-y\| \quad(x ; y \in \mathbf{B}),
$$

with $\phi$ unrestricted except for being nonnegative and $\phi(r, 0, r)=r, r \geq 0$.

These conditions, and others like them, are all equivalent in a Banach space, for each is necessary and sufficient to insure the euclidean character of all subspaces. In a more general environment, however, this is not the case, and so the desirability of making a comparative study of such conditions in more general spaces is suggested. In this note the larger environment is furnished by the class $\{M\}$ of complete, metrically convex and externally convex,

${ }^{1}$ This note deals exclusively with normed linear spaces over the field of reals.

Received November 1, 1953. Presented to the Society, September 5, 1953.

Pacific J. Math. 5 (1955), 161-167 
metric spaces, of which the class of Banach spaces is a very special subclass. $^{2}$ After giving purely metric interpretations of those conditions that we shall discuss (in order that they might be meaningful in spaces of class $\{M\}$ ) we are chiefly concerned with showing that the Jordan-von Neumann relation $(* *)$ characterizes class $\{\mathbf{E}\}$ among the class $\{M\} .^{3}$ This is true, a fortiori, for Fréchet's condition $\left({ }^{*}\right)$ also, but an easy example shows that the inequality used in Schoenberg [10] is not so extensible.

\section{Metrization of the Jordan-von Neumann relation and comparison with other} four-point conditions. Writing $(* *)$ in the form

$$
\left\|\left(p_{1}+p_{2}\right) / 2\right\|=\frac{1}{2}\left[2\left\|p_{1}\right\|^{2}+2\left\|p_{2}\right\|^{2}-\left\|p_{1}-p_{2}\right\|^{2}\right]^{1 / 2}
$$

we see that the length $\left\|\left(p_{1}+p_{2}\right) / 2\right\|$ of the median of the triangle with vertices $\theta, p_{1}, p_{2}(\theta$ denotes the null element of $\mathbf{B})$ is the same function of the lengths $\left\|p_{1}\right\|_{2}\left\|p_{2}\right\|,\left\|p_{1}-p_{2}\right\|$ of the sides of the triangle that it is euclidean space. Since any three elements $x, y, z$ of $\mathbf{B}$ are superposable with $\theta, p_{1}=y-x, p_{2}=z-x$ (the middle-element $(y+z) / 2$ of $y, z$ being carried into $\left(p_{1}+p_{2}\right) / 2$, the middle-element of $\left.p_{1}, p_{2}\right)$ we have the following metric interpretation of (**): $(\dagger)$ every four elements $p, q, r, s$ of $\mathbf{B}$ with $q$ a middleelement of $p, r$ (that is, $p q=q r=p r / 2)$ are congruently imbeddable in the euclidean plane $E_{2}$.

In this formulation, the Jordan-von Neumann criterion is meaningful in every metric space and may, therefore, be compared with other so-called four-point conditions that antedated it.

A metric space has the euclidean $k$-point property provided each $k$-tuple of its elements is congruently contained in a euclidean space (and hence in an $\left.E_{k-1}\right)$. Observing that every metric space has the euclidean three-point property, W. A. Wilson [11] investigated in 1932 the consequences of assuming that a space has the euclidean four-point property. It follows from a result due to the writer $[2, p .131]$ that if $M$ is any metric space whatever, and $M^{1 / 2}$ denotes the space obtained by taking the positive square root of the metric of $M$, then $M^{1 / 2}$ has the euclidean four-point property. Thus the special class $\left\{M^{1 / 2}\right\}$ of spaces with the euclidean four-point property has the same cardinality as the class of

\footnotetext{
${ }^{2}$ For definitions of these and other metric concepts used in this paper see [2].

${ }^{3}$ The abstract of [8] given in Math. Rev. vol. 13 (]952) p. 850 indicates a connection between that paper (which the writer has not seen) and the present note.
} 
all metric spaces (of which it is a proper subclass) and consequently the same is true of the class of all spaces with the euclidean four-point property.

But none of the spaces $\left\{M^{1 / 2}\right\}$ is metrically convex, and Wilson proved that if a complete, metrically convex space has the euclidean four-point property, it is congruent with a subset of a generalized euclidean space. If also external convexity is assumed, then congruence with a generalized euclidean space results.

The weak euclidean four-point property, introduced by the writer in 1933, assumes the congruent imbedding in euclidean space (and hence in $E_{2}$ ) of only those quadruples that contain a linear triple (that is, a triple which is imbeddable in $E_{1}$ ), and it was shown that the weak euclidean four-point property suffices to obtain all of the results that $\mathbb{W}$ ilson had proved by use of the stronger assumption [2, pp.123-128]. But the Jordan-von Neumann condition, as metrized in $(\dagger)$, restricts the class of quadruples assumed to be imbeddable in euclidean space even more than does the weak euclidean four-point property, and consequently is a weaker assumption. We shall refer to it as the feeble euclidean four-point property.

\section{Equivalence in $\{M\}$ of the feeble and the weak euclidean four-point} properties. We prove in this section that in complete, metrically convex and externally convex metric spaces, the feeble euclidean four-point property implies (and hence is equivalent to) the weak euclidean four-point property. Some elementary consequences of the feeble property in such a space are first set down.

I. Middle-elements are unique; for if $p, r \in \mathbf{M}(p \neq r)$ and $q_{1}, q_{2}$ are middleelements of $p, r$ then

$$
p, q_{1}, q_{2}, r \approx p^{\prime}, q_{1}^{\prime}, q_{2}^{\prime}, r^{\prime}
$$

where the "primed" points are in $E_{2}$ and " $\approx$ " denotes the congruence relation. But then $q_{1}^{\prime}$ and $q_{2}^{\prime}$ are middle-points of $p^{\prime}, r^{\prime}$ and consequently

$$
q_{1}^{\prime}=q_{2}^{\prime}, q_{1} q_{2}=q_{1}^{\prime} q_{2}^{\prime}=0, q_{1}=q_{2} \text {. }
$$

II. Each two distinct elements are joined by exactly one metric segment. Since $M$ is complete, metrically convex and metric, each two of its distinct points are joined by at least one metric segment. If $p, r \in \mathbf{M}(p \neq r)$ and $S_{p, r^{*}}$ $S_{p, r}^{*}$ are two segments with end-elements $p, r$, suppose $q^{*}$ belongs to the second 
segment and not to the first. Then $p \neq q^{*} \neq r$, and traversing $S_{p, r}^{*}$ from $q^{*}$ to $p$ a first point $p^{*}$ of $S_{p, r}$ is encountered. Similarly, traversing $S_{p, r}^{*}$ from $q^{*}$ to $r$ a first point $r^{*}$ of $S_{p, r}$ is obtained. The sub-segments $S_{p^{*}, r^{*}}, S_{p^{*}, r^{*}}^{*}$ have only their end-elements in common, but each obviously contains a middle-element of $p^{*}, r^{*}$, contrary to $\mathrm{I}$.

III. Segments admit unique prolongations. Since M is externally convex, each segment may be prolonged beyond its end-elements. But if $S_{p, q}$ admits two prolongations beyond $q$, then clearly elements $r, r^{*}$ of different prolongations exist $\left(r \neq r^{*}\right)$ such that $q$ is a middle-element of $p^{*}, r$ as well as a middleelement of $p^{*}, r^{*}$ for some element $p^{*}$ of $S_{p, q}$. The congruent imbedding in $E_{2}$ of $p^{*}, q, r, r^{*}$ shows this to be impossible.

IV. Each two distinct elements of $M$ are on exactly one metric line. Since $M$ is metric, complete, metrically convex and externally convex, each two of its distinct points $p, q$ are on at least one metric line $L(p, q)[2$, p. 56]. It follows at once from II and III that $L(p, q)$ is unique.

THEOREM 3.1. If $p$ is a point and $L$ a metric line of $M$, then $L+(p)$ is congruently imbeddable in $E_{2}$.

Proof. If $p \in L$ then

$$
L+(p)=L \approx E_{1} \subset E_{2},
$$

by the definition of a metric line. Suppose $p \notin L$, select points $r_{0}, r_{1}$ on $L$ with $r_{0} r_{1}=1$, and let $p^{\prime}, r_{0}^{\prime}, r_{1}^{\prime}$ be points of $E_{2}$ such that $p, r_{0}, r_{1} \approx p^{\prime}, r_{0}^{\prime}, r_{1}^{\prime}$. Let $L^{\prime}$ denote the straight line of $E_{2}$ determined by $r_{0}^{\prime}, r_{1}^{\prime}$, and consider the one-toone correspondence

$$
\Gamma: p \leftrightarrow p^{\prime}, L\left(r_{0}, r_{1}\right) \approx L^{\prime}\left(r_{0}^{\prime}, r_{1}^{\prime}\right),
$$

where the congruence of the two lines is the unique extension of the congruence $r_{0}, r_{1} \approx r_{0}^{\prime}, r_{1}^{\prime}$. We shall show that $\Gamma$ is a congruence.

If $r_{1 / 2}$ denotes the unique middle-point of $r_{0}, r_{1}$, and $r_{1 / 2}^{\prime}=\Gamma\left(r_{1 / 2}\right)$, then $r_{1 / 2}^{\prime}$ is the middle-point of $r_{0}^{\prime}, r_{1}^{\prime}$. By the feeble euclidean four-point property

$$
p, r_{0}, r_{1 / 2}, r_{1} \approx \bar{p}, \bar{r}_{0}, \bar{r}_{1 / 2}, \bar{r}_{1}
$$

with the "barred" points in $E_{2}$, and since $p^{\prime}, r_{0}^{\prime}, r_{1}^{\prime} \approx p, r_{0}, r_{1}$, a motion of $E_{2}$ exists that carries $p, r_{0}, r_{1}$ into $p^{\prime}, r_{0}^{\prime}, r_{1}^{\prime}$, respectively. This motion evidently 
sends $\bar{r}_{1 / 2}$ into $r_{1 / 2}^{\prime}$, and we have

$$
p, r_{0}, r_{1 / 2}, r_{1} \approx p^{\prime}, r_{0}^{\prime}, r_{1 / 2}^{\prime}, r_{1}^{\prime}
$$

that is, $p r_{1 / 2}=p^{\prime} r_{1 / 2}^{\prime}$.

If $r_{3 / 4}$ denotes the middle-point of $r_{1 / 2}, r_{1}$, and $r_{3 / 4}^{\prime}=\Gamma\left(r_{3 / 4}\right)$, the feeble fourpoint property, applied to the quadruple $p, r_{\frac{1}{2},}, r_{3 / 4}, r_{1}$ gives $p r_{3 / 4}=p^{\prime} r_{\frac{3}{4}}^{\prime}$. Continuing in this manner, we obtain $p r_{i / 2 n}=p^{\prime} r_{i / 2^{n}}^{\prime}$ for each dyadically rational fraction $i / 2^{n}$. Since the points $r_{i / 2^{n}}$ are dense in $\operatorname{seg}\left[r_{0}, r_{1}\right]$, continuity of the metric (and continuity of the congruence $L\left(r_{0}, r_{1}\right) \approx L^{\prime}\left(r_{0}^{\prime}, r_{1}^{\prime}\right)$ ) yields $p x=p^{\prime} x^{\prime}, x^{\prime}=\Gamma(x)$, for every $x \in \operatorname{seg}\left[r_{0}, r_{1}\right]$.

Let $r_{2}$ be a point of $L$ such that $r_{1}$ is the middle-point of $r_{0}, r_{2}$. The feeble four-point property gives (in the manner employed above) $p, r_{0}, r_{1}, r_{2} \approx p^{\prime}, r_{0}^{\prime}, r_{1}^{\prime}, r_{2}^{\prime}$, where $r_{2}^{\prime}=\Gamma\left(r_{2}\right)$, and consequently $p r_{2}=p^{\prime} r_{2}^{\prime}$. Then from $p, r_{1}, r_{2} \approx p^{\prime}, r_{1}^{\prime}, r_{2}^{\prime}$ we obtain $p x=p^{\prime} x^{\prime}, x \in \operatorname{seg}\left[r_{1}, r_{2}\right]$ in the same manner as described above for $\operatorname{seg}\left[r_{0}, r_{1}\right]$. It is clear that a continuation of the procedure establishes $p x=p^{\prime} x^{\prime}$ for every $x$ of $L$ and $x^{\prime}=\Gamma(x)$.

THEOREM 3.2. In a complete, metrically convex and externally convex metric space $\mathrm{M}$, the feeble and the weak euclidean four-point properties are equivalent.

Proof. The weak property obviously implies the feeble one in any metric space. Suppose $M$ has the feeble property, and $p, q, r, s \in M$ (pairwise distinct) with $q, r, s$ congruent with a triple of $E_{1}$. Then the line $L(q, r)$ contains $s$, and $L(q, r)+(p)$ is congruently imbeddable in $E_{2}$. Hence $p, q, r, s$ are imbeddable in $E_{2}$.

4. Extension of the Jordan-von Neumann theorem. The writer has shown $[2, \mathrm{p} .127]$ that a complete, metrically convex and externally convex semimetric space with the weak euclidean four-point property has the euclidean $k$-point property for every positive integer $k$. It follows easily that such a space is generalized euclidean. Use of Theorem 3.2 now yields the following result:

THEOREM 4.1. A complete, metrically convex and externally convex metric space with the feeble euclidean four-point property is generalized euclidean.

This is the desired extension of the Jordan-von Neumann theorem for real normed linear spaces. For if $\mathbf{L}$ is such a space, and $\mathbf{L}$ satisfies the Jordanvon Neumann condition $\left({ }^{* *}\right)$, then the Banach space that arises by completing 
$\mathbf{L}$ in the Hausdorff manner is a complete, metrically convex and externally convex metric space with the feeble euclidean four-point property. According to Theorem 4.1, it is generalized euclidean and so an inner product is definable in it. Hence an inner product is definable in $\mathbf{L}$, and the Jordan-von Neumann theorem for real normed linear spaces is obtained. Thus the metric essence of $(* *)$ determines the euclidean character of $\mathbf{L}$ by use of the purely metric features of the space, without regard, for example, for its very special properties due to linearity.

5. Concluding remarks. Condition $(*)$ of Fréchet is equivalent to Wilson's euclidean four-point condition [2, p. 106] and consequently his theorem of 1935 had already been proved in more general form by Wilson in 1932 .

A semimetric space is ptolemaic provided for any four of its elements $p$, $q, r, s$, the three products $p q \cdot r s, p s \cdot q r, p r \cdot q s$ of "opposite" distances satisfy the triangle inequality. Schoenberg [10] showed that in a real linear seminormed ptolemaic space, the semi-norm satisfies the triangle inequality (and so is actually a norm) and an inner product is definable which is related to the norm in the usual way.

Schoenberg's ptolemaic condition which (as a norm postulate in $L$ has the form

$$
\|f\| \cdot\|g-h\|+\|g\| \cdot\|h-f\| \geq\|h\| \cdot\|f-g\| \quad(f, g, h \in \mathbf{L})
$$

is not extensible to the class $\{M\}$. For if three pairwise distinct rays of $E_{2}$, with a common initial point, be metrized convexly (that is, if $p, q$ are points of different rays, then $p q=e(p, o)+e(o, q)$, where $e($,$) denotes euclidean$ distance and $o$ is the common point of the rays, while $p q=e(p, q)$ if $p, q$ belong to the same ray) the resulting space is easily shown to be metric, complete, convex and externally convex, and ptolemaic. But it is not, of course, generalized euclidean. It would be interesting to know whether or not this "tripod" is present in every such example.

\section{REFERENCES}

1. N. Aronszajn, Caracteŕisation métrique de l'espace de Hilbert, des espaces vectoriels et de certains groupes métriques, C. R. Acad. Sci. Paris, 201 (1935), 811-813.

2. L. M. Blumenthal, Theory and applications of distance geometry, Oxford, at the Clarendon Press, 1953.

3. M.M. Day, Some characterizations of inner product spaces, Trans. Amer. Math. Soc. 62 (1947), $320-337$. 
4. F.A. Ficken, Note on the existence of scalar products in normed linear spaces, Ann. of Math. 45 (1944), $362-366$.

5. M. Fréchet, Sur la définition axiomatique d'une classe d'espaces vectoriels distanciés applicables vectoriellement sur l'espace de Hilbert, Ann. of Math. 36 (1935), $705-718$.

6. P. Jordan and J. von Neumann, On inner products in linear metric spaces, Ann. of Math. 36 (1935), 719-723.

7. M. Nagumo, Characterisierung der allgemeine euklidischen Räume durch ein Postulat für Schwerpunkte, Jap. Jour. of Math. 12 (1936), 123-128.

8. K. Ohira, On a certain complete, separable and metric space, Mem. Fac. Sci. Kyūsyū Univ. A 6 (1951), 9-15.

9. H. Rubin and M. Stone, Postulates for generalizations of Hilbert space, Proc. Amer. Math. Soc. 4 (1953), 611-616.

10. I. J. Schoenberg, $A$ remark on M. M. Day's characterization of inner product spaces and a conjecture of L.M. Blumenthal, Proc. Amer. Math. Soc. 3 (1952), 961-964.

11. W. A. Wilson, A relation between metric and euclidean spaces, Amer. Jour. Math. 54 (1932), 505-517.

UNIVERSITY OF MISSOURI 



\section{PACIFIC JOURNAL OF MATHEMATICS}

\section{EDITORS}

\author{
H. L. ROYDEN \\ Stanford University \\ Stanford, California \\ E. Hewitt \\ University of Washington \\ Seattle 5 , Washington
}

R. P. Dilworth

California Institute of Technology Pasadena 4, California

A. HorN*

University of California

Los Angeles 24, California

\section{ASSOCIATE EDITORS}

\author{
H. BUSEMANN \\ HERBERT FEDERER \\ MARSHALL HALL
}

\author{
P. R. HALMOS \\ HEINZ HOPF
}

ALFRED HORN
R. D. JAMES

BORGE JESSEN

PAUL LÉVY
GEORGE PÓLYA

J. J. STOKER

KOSAKU YOSIDA

\section{SPONSORS}

UNIVERSITY OF BRITISH COLUMBIA

CALIFORNIA INSTITUTE OF TECHNOLOGY

UNIVERSITY OF CALIFORNIA, BERKELEY

UNIVERSITY OF CALIFORNIA, DAVIS

UNIVERSITY OF CALIFORNIA, LOS ANGELES

UNIVERSITY OF CALIFORNIA, SANTA BARBARA

MONTANA STATE UNIVERSITY

UNIVERSITY OF NEVADA

OREGON STATE COLLEGE

UNIVERSITY OF OREGON

UNIVERSITY OF SOUTHERN CALIFORNIA

\author{
STANFORD RESEARCH INSTITUTE \\ STANFORD UNIVERSITY \\ UNIVERSITY OF UTAH \\ WASHINGTON STATE COLLEGE \\ UNIVERSITY OF WASHINGTON
}

AMERICAN MATHEMATICAL SOCIETY HUGHES AIRCRAFT COMPANY SHELL DEVELOPMENT COMPANY

Mathematical papers intended for publication in the Pacific Journal of Mathematics should be typewritten (double spaced), and the author should keep a complete copy. Manuscripts may be sent to any of the editors. Manuscripts intended for the outgoing editors should be sent to their successors. All other communications to the editors should be addressed to the managing editor, Alfred Horn at the University of California, Los Angeles 24, California.

50 reprints of each article are furnished free of charge; additional copies may be obtained at cost in multiples of 50 .

The Pacific Journal of Mathematics is published quarterly, in March, June, September, and December. The price per volume (4 numbers) is $\$ 12.00$; single issues, $\$ 3.50$. Back numbers are available. Special price to individual faculty members of supporting institutions and to individual members of the American Mathematical Society: $\$ 4.00$ per volume; single issues, $\$ 1.25$.

Subscriptions, orders for back numbers, and changes of address should be sent to Pacific Journal of Mathematics, c/o University of California Press, Berkeley 4, California.

Printed at Kokusai Bunken Insatsusha (International Academic Printing Co., Ltd.), No. 10, 1-chome, Fujimi-cho, Chiyoda-ku, Tokyo, Japan.

* During the absence of E. G. Straus.

PUBLISHED BY PACIFIC JOURNAL OF MATHEMATICS, A NON-PROFIT CORPORATION COPYRIGHT 1955 BY PACIFIC JOURNAL OF MATHEMATICS 


\section{Pacific Journal of Mathematics}

\section{Vol. 5, No. $2 \quad$ October, 1955}

Leonard M. Blumenthal, An extension of a theorem of Jordan and von

Neumann ........................................ 161

L. Carlitz, Note on the multiplication formulas for the Jacobi elliptic functions.......................................... 169

L. Carlitz, The number of solutions of certain types of equations in a finite

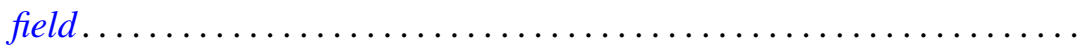

George Bernard Dantzig, Alexander Orden and Philip Wolfe, The generalized simplex method for minimizing a linear form under linear

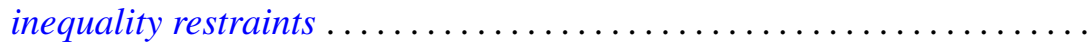

Arthur Pentland Dempster and Seymour Schuster, Constructions for poles and polars in n-dimensions . . . . . . . . . . . . . . . . . . . . 197

Franklin Haimo, Power-type endomorphisms of some class 2 groups ...... 201

Lloyd Kenneth Jackson, On generalized subharmonic functions ......... 215

Samuel Karlin, On the renewal equation ...................... 229

Frank R. Olson, Some determinants involving Bernoulli and Euler numbers of higher order................................ 259

R. S. Phillips, The adjoint semi-group ........................ 269

Alfred Tarski, A lattice-theoretical fixpoint theorem and its applications ... 285

Anne C. Davis, A characterization of complete lattices .............. 311 